\title{
Colorectal cancer in South Africa: An assessment of disease presentation, treatment pathways and 5-year survival
}

\author{
M Brand, ${ }^{1}$ FCS (SA), PhD, Cert Surgical Gastroenterology (SA); P Gaylard, ${ }^{2}$ PhD; J Ramos, ${ }^{3}$ MB BCh, FCS (SA) \\ ${ }^{1}$ Department of Surgery, Faculty of Health Sciences, University of Pretoria, South Africa; School of Physiology, Faculty of Health Sciences, \\ University of the Witwatersrand, Johannesburg, South Africa \\ ${ }^{2}$ Data Management and Statistical Analysis, Johannesburg, South Africa \\ ${ }^{3}$ Wits Donald Gordon Medical Centre, Johannesburg, South Africa; Department of Surgery, Faculty of Health Sciences, University of the \\ Witwatersrand, Johannesburg, South Africa
}

Corresponding author: M Brand (martinbrand78@gmail.com)

Background. Colorectal cancer (CRC) is the fourth most common cancer in South Africa (SA), and the sixth most lethal. Approximately $25 \%$ of patients will have synchronous metastatic disease at the time of their primary CRC diagnosis. Although chemotherapy is used in most stages of the disease, surgical resection of the primary tumour and metastases remains the most successful treatment modality to achieve cure or prolong survival. To date, no data on CRC presentation and management have been published in SA.

Objectives. To determine CRC presentation, general management patterns and overall survival in the SA private healthcare sector.

Methods. A retrospective review of a private healthcare funder's database from 1 January 2008 to 31 December 2015. International Statistical Classification of Diseases and Related Health Problems (10th revision) (ICD-10) diagnosis codes were used to identify colorectal cancer and liver and/or pulmonary metastatic disease. Procedure codes assigned to hospital admissions were used to identify type of surgical treatment. Chemotherapy was identified by the World Health Organization Anatomical Therapeutic Chemical Classification System of medicines. Treatment patterns were determined and 5-year survival rates for these were calculated. Survival was estimated using the Kaplan-Meier method, and Cox proportional hazards regression was used for between-group comparisons of survival. Data analysis was carried out using SAS version 9.4 for Windows.

Results. A total of 3412 patients were included in the study, 2267 with CRC only and 1145 with liver (LM) or pulmonary metastases (PM). The mean age was 64.1 years (range 21 - 97), and 54.6\% were male; these did not differ statistically between the study groups. Twenty percent of patients with LM or PM underwent surgical resection of their metastases. Five-year survival rates following surgical resection of all disease for CRC only, CRCLM, CRCPM and CRCLMPM were 71.7\%, 57.3\%, 31.5\% and 26.0\%, respectively.

Conclusions. SA CRC patients treated in the private healthcare sector have similar disease presentation to that in published international series, with similar outcomes following various treatment pathways; however, it seems that fewer resections of metastases are undertaken compared with international trends.

S Afr Med J 2018;108(2):118-122. DOI:10.7196/SAMJ.2018.v108i2.12338

In South Africa (SA), colorectal cancer (CRC) is the fourth most common cancer among both men and women. The crude incidence is 7.17/100 000/year for men and 5.80/100 000/year for women, ${ }^{[1]}$ and CRC ranks sixth in cancer-related mortality. ${ }^{[2]}$

The cumulative lifetime risk of developing CRC in SA is 1.24 for males and 0.74 for females. ${ }^{[1]}$ At the time of CRC diagnosis, $20-25 \%$ of patients will have metastatic disease, ${ }^{[3]}$ the liver being the most common site. ${ }^{[4]}$ More than half of the patients who undergo curative resection may expect to have tumour recurrence, either locally or as metastatic disease. ${ }^{[5]}$ CRC therefore represents a significant healthcare burden.

Five-year overall survival rates for CRC are 93.2\% for stage I, 72.2 $84.7 \%$ for stage II, $52.3-83.4 \%$ for stage III and $8.1 \%$ for stage IV ${ }^{[6]}$ Stage IV disease treated with chemotherapy alone has a median overall survival of 12 months. ${ }^{[7]}$ Various treatment algorithms have been incorporated into international guidelines, but common to most is that, when possible, surgical resection of the primary lesion and its metastases offers the best survival benefit for patients. ${ }^{[8]}$ So far, a specific treatment algorithm tailored to SA patients has not been published.

\section{Objectives}

To describe the presentation of CRC patients in a privately insured population in SA, general clinical management trends, and 5-year overall survival for various disease stages.

\section{Methods \\ Study design and data collection}

A retrospective review of a private healthcare funder's database from 1 January 2008 to 31 December 2015 was undertaken. Ethics approval was granted by the University of the Witwatersrand Human Research Ethics Committee (ref. no. M141027), and consent to obtain deidentified data was given by the principal officer of the private healthcare funder. The initial dataset was based on claims by and clinical information on active members of the medical scheme. Data were extracted from tables developed by the medical scheme for analysis where the table development included data validation. Unique entities were identified from these data tables of members with a validated CRC diagnosis.

Patients included in this study were enrolled on the scheme's oncology benefit for the treatment of CRC. International Statistical 
Classification of Diseases and Related Health Problems (10th revision) (ICD-10) diagnosis codes were used to identify CRC only, with or without liver and/or pulmonary metastatic disease. Once identified, secondary or unspecified malignant neoplasms of the digestive organs or respiratory tract were sought for in this patient population. The authors examined each identified record to determine whether or not the additional diagnosis was relevant to the initial CRC diagnosis. Sites of metastases other than the liver and lungs were excluded.

All procedure codes assigned to hospital admissions were captured, and each code was examined by the authors to identify surgical treatments relevant to resections of CRC and CRC metastases. All other procedures were excluded. Chemotherapy was identified by the World Health Organization Anatomical Therapeutic Chemical Classification System of medicines. The number of days from diagnosis of CRC and/or CRC metastases and from surgical resection were collected. Death was based on date of withdrawal from the medical scheme, and survival on ongoing contribution to the scheme. Patients were excluded if they were aged $<18$ years or were diagnosed before 1 January 2008, and if they were diagnosed on or after 1 January 2014 to ensure that we did not miss any treatment modalities and that patients had at least 1 year of follow-up.

Each patient's treatment was assessed as surgical resection of the cancer with or without chemotherapy, chemotherapy without surgical resection, or no treatment (no surgical resection or chemotherapy). Four patient groups were used for analysis: (i) CRC only; (ii) CRC and liver metastases (CRCLM); (iii) CRC and pulmonary metastases (CRCPM); and (iv) CRC, liver and pulmonary metastases (CRCLMPM).

\section{Statistical analysis}

Crude incidence was calculated and represented as 100 000/ year. Categorical variables were summarised as percentages, and continuous variables were summarised as means, standard deviations (SDs), medians and interquartile ranges. Survival was estimated using the Kaplan-Meier method and Cox proportional hazards regression was used for between-group comparisons of survival. The 5\% significance level was used. Data analysis was carried out using SAS version 9.4 for Windows (SAS Institute, USA).

\section{Results}

A total of 3412 patients were included in the study (Fig. 1). Their demographic features are set out in Table 1 . The total number of members on 1 January 2015 was 2658 100, with 5093 diagnosed with CRC. The crude incidence of CRC in this insured population increased from $18.9 / 100000$ in 2008 to $21.3 / 100000$ in 2015. The gender composition for each patient group was similar, apart from the proportion of males being significantly higher in the CRCLM group compared with the CRC-only group $(p=0.037$ ) (Table 1). The mean age of the CRC-only group (64.1 years, SD 12.8) was significantly higher than that of the CRCLMPM group (61.5 years, SD 11.7) $(p<0.0001)$. There was no statistically significant difference between the CRCLM, CRCPM and CRCLMPM groups (Table 1).
Disease presentation, proportions of cancer with or without metastases, and management pathways of the study group are presented in Fig. 2. One-third of patients either presented with or developed LM and/or PM, the most common being LM. Two-thirds of these patients underwent surgical resection, but only $7.2 \%$ (83/1 145) of patients with metastatic disease underwent metastatectomy. The most common was resection of LM, but only $10.0 \%$ (60/601) of patients with LM underwent liver resection; of these, 6.7\% (4/60) underwent more than one liver resection. Of patients with CRC only, the majority underwent resection of their primary lesion. No treatment was recorded by the medical aid scheme for $16.5 \%$ of patients.

Fig. 3 depicts overall survival regardless of therapy. Using the CRConly group as reference, the risk of death was higher for all the other three cancer groups, and highest for CRCLMPM. Five-year overall survival (5YOS) for CRCPM was better than for CRCLM.

Table 2 describes treatment-related 5 -year survival. The best $5 Y O S$ was for CRC without metastatic disease treated by surgical resection and chemotherapy. For patients with metastatic disease, CRCLM resection preceded by chemotherapy provided the best $5 Y O S$ of $40.7 \%$. CRCPM resection with chemotherapy had 5 YOS of $31.7 \%$. There were 5 -year overall survivors who did not have surgical

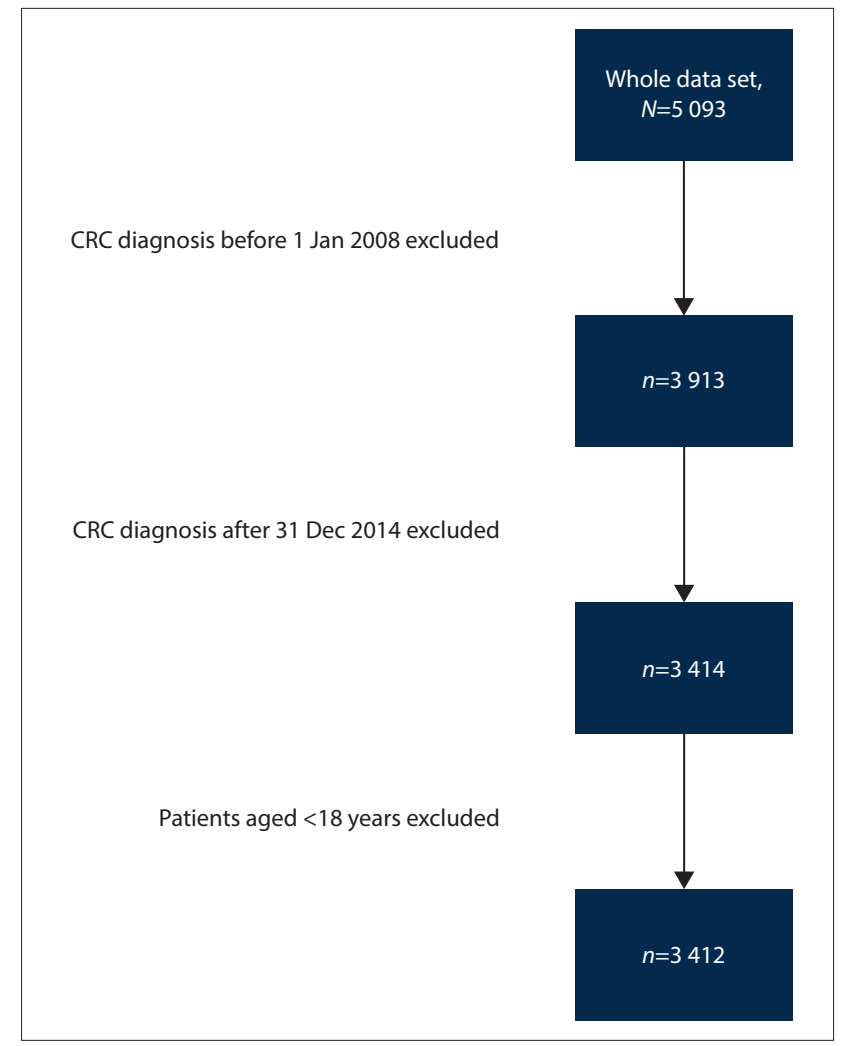

Fig. 1. Data selection, 1 January 2008 - 31 December 2015. $(C R C=$ colorectal cancer.)

\begin{tabular}{|c|c|c|c|c|c|}
\hline & All cases & CRC only & CRCLM & CRCPM & CRCLMPM \\
\hline Survival, $n(\%)$ & 3412 & $2267(66.4)$ & $601(17.6)$ & $182(5.3)$ & $362(10.6)$ \\
\hline Age (yr), mean (range) & $64.1(21-97)$ & $64.7(21-97)$ & $63.6(25-93)$ & $62.6(23-85)$ & $61.5(23-87)$ \\
\hline \multicolumn{6}{|l|}{ Gender, $n(\%)$} \\
\hline Males & $1862(54.6)$ & $1203(53.1)$ & $357(59.4)$ & $97(53.3)$ & $205(56.6)$ \\
\hline Females & $1550(45.4)$ & $1064(46.9)$ & $244(40.6)$ & $85(46.7)$ & $157(43.4)$ \\
\hline
\end{tabular}




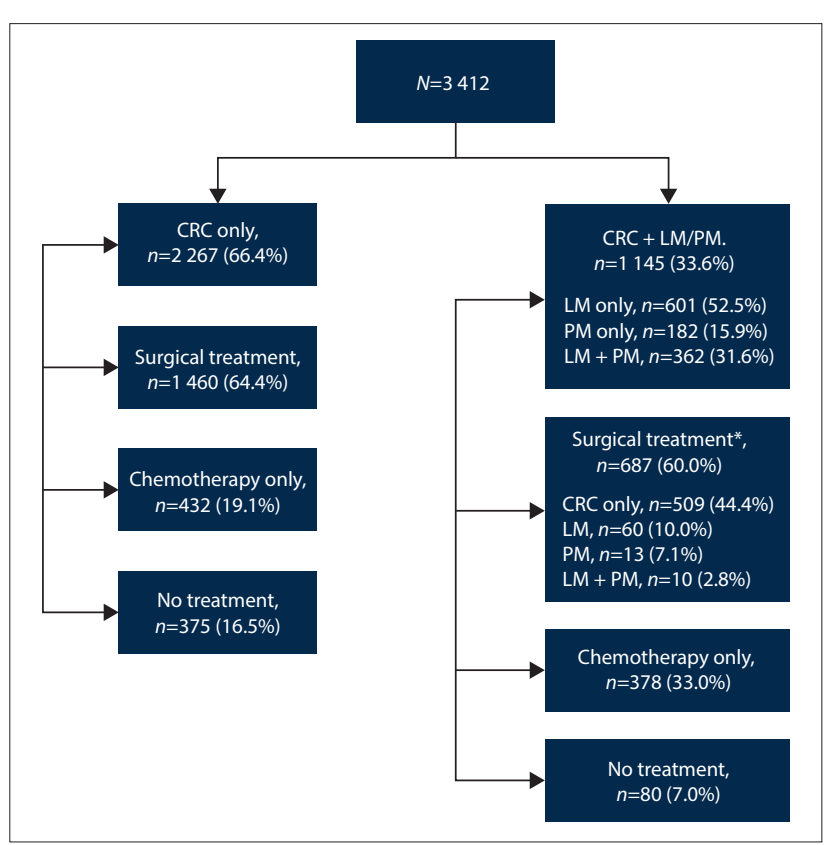

Fig. 2. Disease presentation and management. $(C R C=$ colorectal cancer; $L M=$ liver metastases; $P M=$ pulmonary metastases. ${ }^{*}$ Patients may or may not have received chemotherapy.)

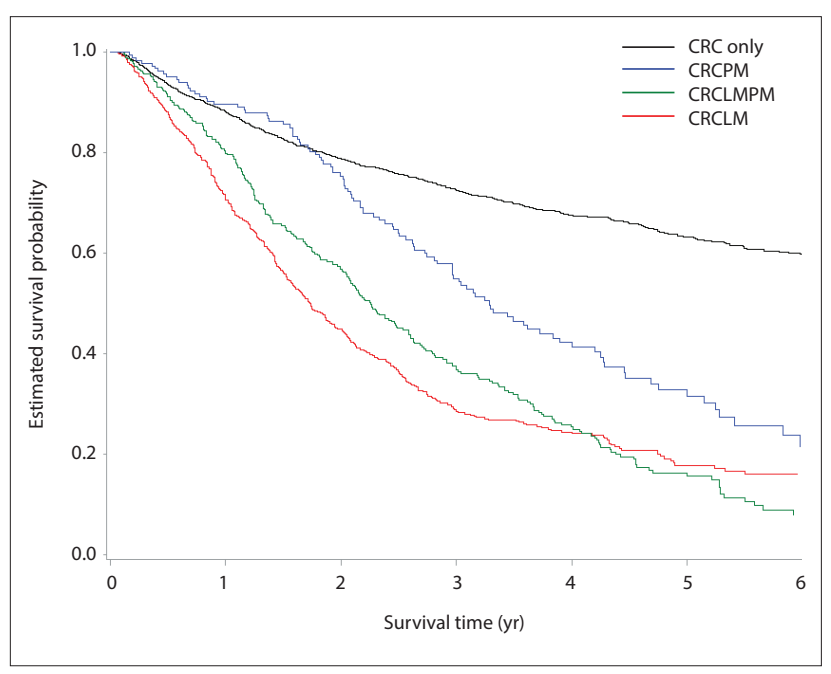

Fig. 3. Overall survival of disease groups, regardless of treatment. (CRC = colorectal cancer; $L M=$ liver metastases; $P M=$ pulmonary metastases.)

resection of metastases, but the figure was significantly lower than if they had had all their disease resected.

\section{Discussion}

This is the first study to determine survival outcomes of CRC with or without LM and/or PM in a selected, privately insured SA population. We have demonstrated that one-third of patients with CRC present with or develop LM and/or PM, of whom 7\% undergo surgical metastatectomy. $5 \mathrm{YOS}$ is best for patients with disease isolated to the colon or rectum that is resected and treated with chemotherapy. Furthermore, there is improved survival with surgical resection in combination with chemotherapy of LM and/or PM.

\section{Incidence and presentation of CRC}

The global incidence rates of CRC, overall and in males and females, are $17.2 / 100000,20.6 / 100000$ and $14.3 / 100000$, respectively. The incidence rates of CRC in Europe, overall and in males and females, are 29.5/100 000, 37.3/100 000 and 23.6/100 000, respectively. ${ }^{[1]}$ The corresponding figures for sub-Saharan Africa are 5.8/100 000, 6.4/100 000 and 5.4/100 000, respectively. ${ }^{[1]}$ The incidence in this study falls in between that for developed countries and African statistics, possibly indicating that screening and surveillance of CRC are better than in developing countries but may not be as stringent as in developed countries.

\section{Management and overall survival of CRC groups CRC only}

In our study, patients who received chemotherapy either before or after resection of the primary CRC appeared to have improved survival compared with those who underwent surgical resection only (Table 2). CRC treatment has been shown to be more effective when surgery is combined with chemotherapy. ${ }^{[9]}$ The long survival of the chemotherapy-only group in this study is not in keeping with international series, and cannot be explained owing to the limited available clinical information such as accurate tumour staging.

\section{CRCLM}

Complete resection of CRCLM is the only treatment associated with long-term survival, with 37 - 54\% 5YOS following surgical resection as opposed to 4 - $9 \%$ without surgical resection, ${ }^{[10]}$ and potential cure in up to $16 \%$ of patients. ${ }^{[11]}$

In this series, 5 YOS following resection of all disease was $57.3 \%$ if chemotherapy was administered before tumour resection, and $40.7 \%$ if surgery preceded chemotherapy (Table 2). Synchronous resection of the primary lesion as well as the liver metastases has been reported to have $40-47 \% 5$ YOS. $^{[12]}$ We were not able to determine 5 YOS for synchronous resection in this study.

CRCLM resection is safe, with a mortality rate of $<2 \%$ and a major morbidity rate of $\sim 12.5 \%,{ }^{[13,14]}$ including patients aged $>75$ years, who can achieve 5 YOS of $58 \% \cdot{ }^{[15]}$ Patients may undergo repeated liver resections for recurrent CRCLM with similar survival figures to patients who only have one liver resection and no recurrence. One case series reported $47 \% 5$ YOS for repeat liver resection. ${ }^{[10]}$ We were not able to perform survival analyses for repeated resections owing to the low numbers of these procedures.

\section{CRCPM}

Since the more widespread use of chemotherapy for pulmonary CRC metastases, overall survival has improved ${ }^{[16]} \mathrm{PM}$ are typically single or multiple nodules, as opposed to a miliary type of spread or lymphangitis carcinomatosa, and hence more amenable to surgical resection. ${ }^{[17]}$ 5 YOS following pulmonary metastectomy has been reported as 24 $61.4 \%,{ }^{[18]}$ and in this study was $31.5 \%$ (Table 2 ). The CRCPM resection mortality rate is $<1 \%$ and the morbidity rate is $\sim 14 \%{ }^{[19]}$

\section{CRCLMPM}

5 YOS for patients with pulmonary metastectomy following hepatic metastectomy is $31.6 \% \cdot{ }^{[17]}$ In this study, 5 YOS was $26.0 \%$ (Table 2). Our figures also highlight the fact that relatively few patients undergo surgical resection of their metastatic disease. Median overall survival has been shown to be significantly improved with complete or macroscopically clear resection margins of CRCLMPM compared with palliative chemotherapy, ${ }^{[20]}$ as well as resection of the primary CRC only in stage IV disease. ${ }^{[21]}$

\section{Study limitations}

There are limitations to this study. Data were retrieved from a single private healthcare funder's database which represents $53 \%$ of the 


\begin{tabular}{llc} 
Table 2. Five-year overall survival according to treatment pattern & & \\
\hline Group & Survival, $\boldsymbol{n}$ (\%) & $\mathbf{9 5 \%}$ CI \\
\hline CRC only & & $54.2-66.5$ \\
$\quad$ Surgery only & $489(60.1)$ & $67.6-75.3$ \\
Surgery and chemotherapy & $886(71.7)$ & $56.1-80.1$ \\
Chemotherapy upfront followed by surgery & $85(69.9)$ & $50.5-61.6$ \\
Chemotherapy only, no surgical resection & $432(56.3)$ & $30.5-77.0$ \\
CRCLM & & $20.0-60.5$ \\
Chemotherapy upfront followed by surgical resection of all cancer & $19(57.3)$ & $9.9-22.4$ \\
CRC surgery followed by chemotherapy and further resections & $41(40.7)$ & $5.6-15.5$ \\
CRC surgery only and chemotherapy, no metastases resected & $23(15.6)$ & $23.4-40.0$ \\
Chemotherapy only, no surgical resection & $180(9.8)$ & $12.2-40.9$ \\
CRCPM & $13(31.5)$ & $11.8-35.2$ \\
Chemotherapy and resection of all cancer & $62(25.4)$ & $8.5-47.9$ \\
CRC surgery only and chemotherapy, no metastases resected & $65(22.4)$ & $6.8-21.6$ \\
Chemotherapy only, no surgical resection & & $1.6-12.4$ \\
CRCLMPM & $10(26.0)$ & $101(13.1)$ \\
Chemotherapy and resection of all cancer & $133(5.3)$ &
\end{tabular}

open market. This database is primarily designed for health economic data capture with limited clinical information. These results are based on treatments claimed from the medical scheme. Members may not necessarily submit all treatment claims to the scheme; for example, they may receive treatment as part of a clinical trial, use self-funded medication, or receive treatment from state facilities. Because of the paucity of clinical information, only general treatment patterns are reported. The survival estimates and Kaplan-Meier plot are limited by the fact that the analysed data do not originate from primary clinical data, but from secondary medical aid administrative and claims data. There may therefore be loss of accuracy and missing information. Furthermore, we could not adjust for covariates. However, we believe that the Kaplan-Meier plot remains the best way to display survival data, and we capped the estimates to 5 years, beyond which there are too few patients at risk for reliable estimates. We have provided $95 \%$ confidence intervals for these estimates.

Whether CRC in SA differs from internationally published series or whether patients are being managed appropriately cannot be determined from this study. Furthermore, our results cannot be extrapolated to the public healthcare sector, as the treatment of this disease appears to differ significantly with regard to disease presentation as well as access to chemotherapy and biological agents.

\section{Future research}

Appropriate clinical information should be collected by private healthcare funders to facilitate audit as well as to understand clinical decision-making and outcomes. The importance of multidisciplinary teams (MDTs) cannot be over-emphasised in the assessment of CRC with metastases to determine optimal patient management. A prospective study of an MDT showed that despite $84 \%$ of clinicians being certain of their management plan, following the MDT discussion 36\% changed their management plan; $72 \%$ of these changes were major ones. ${ }^{[22]}$ Furthermore, studies have shown lower survival rates following non-adherence to MDT decisions. ${ }^{[23]}$ Finally, a national CRC registry should be established so that understanding of disease epidemiology, presentation, outcomes and therapeutic resources can be improved.

\section{Conclusions}

CRC patients treated in the SA private healthcare sector have improved survival following resection of the primary tumour and metastatic disease, which is further enhanced by the use of chemotherapy as opposed to chemotherapy alone or no treatment.

Acknowledgements. The authors thank Discovery Health, specifically the Clinical Policy Unit, for their immense assistance with data extraction and management.

Author contributions. MB: conceived the study idea, accessed the data, cowrote the manuscript; PG: performed the statistical analysis, co-wrote the manuscript; JR: contributed to the study idea, co-wrote the manuscript.

Funding. Wits Donald Gordon Medical Centre Research Grant. The statistical analysis of the data was also funded by the Wits Donald Gordon Medical Centre.

Conflicts of interest. None.

1. Cancer Association of South Africa. 2012 National Cancer Registry. http://www.cansa.org.za/ files/2017/03/SA-National-Cancer-Registry-2012-web-Feb-2017.pdf (accessed 8 April 2017).

2. International Agency for Research on Cancer. GLOBOCAN 2012: Estimated cancer incidence, mortality and prevalence worldwide in 2012. http://globocan.iarc.fr/Default.aspx (accessed 16 December 2016).

3. Dervenis C, Xynos E, Sotiropoulos G, et al. Clinical practice guidelines for the management of metastatic colorectal cancer: A consensus statement of the Hellenic Society of Medical Oncologists (HeSMO). Ann Gastroenterol 2016;29(4):390-416. https://doi.org/10.20524/aog.2016.0050

4. Nordlinger B, van Cutsem E, Rougier P, et al. Does chemotherapy prior to liver resection increase the potential for cure in patients with metastatic colorectal cancer? A report from the European Colorectal Metastases Treatment Group. Eur J Cancer 2007;43(14):2037-2045. https://doi.org/10.1016/j. Metastases Treatment Group. Eur J Cancer 2007;43(14):2037-2045. https://doi.org/10.1016/j.
ejca.2007.07.017

5. August DA, Ottow RT, Sugarbaker PH. Clinical perspective of human colorectal cancer metastasis. Cancer Metastasis Rev 1984;3(4):303-324. https://doi.org/10.1007/BF00051457

6. O'Connell JB, Maggard MA, Ko CY. Colon cancer survival rates with the new American Joint Committee on Cancer sixth edition staging. J Natl Cancer Inst 2004;96(19):1420-1425. https://doi. org/10.1093/jnci/djh275

7. Stintzing S. Management of colorectal cancer. F1000Prime Rep 2014;6:108. https://doi.org/10.12703/ P6-108

8. Van Cutsem E, Cervantes A, Nordlinger B, Arnold D; ESMO Guidelines Working Group. Metastatic colorectal cancer: ESMO Clinical Practice Guidelines for diagnosis, treatment and follow-up. Ann Oncol 2014;25(Suppl 3):iiil-iii9. https://doi.org/10.1093/annonc/mdu260

9. Nishioka Y, Moriyama J, Matoba S, et al. Prognostic impact of adjuvant chemotherapy after hepatic resection for synchronous and early metachronous colorectal liver metastases. Dig Surg 2017 (epub 19
reforic resection for synchronous and early metachronous
August 2017). https://doi.org $/ 10.1159 / 000478791$

10. Lemke J, Cammerer G, Ganser J, et al. Survival and prognostic factors of colorectal liver metastases after surgical and nonsurgical treatment. Clin Colorectal Cancer 2016;15(4):e183-e192. https://doi. org/10.1016/j.clcc.2016.04.007 
11. Tomlinson J, Jarnagin W, DeMatteo R, et al. Actual 10-year survival after resection of colorectal liver metastases. J Clin Oncol 2007;25(29):4575-4582. https://doi.org/10.1200/JCO.2007.11.0833

12. Adam R, de Gramont A, Figueras J, et al. of the EGOSLIM (Expert Group on OncoSurgery management of LIver Metastases) group. Managing synchronous liver metastases from colorectal cancer: A multidisciplinary international consensus. Cancer Treat Rev 2015;41(9):729-741. https://doi. org/10.1016/j.ctrv.2015.06.006

13. Schiffman SC, Kim KH, Tsung A, Marsh JW, Geller DA. Laparoscopic versus open liver resection for metastatic colorectal cancer: A meta-analysis of 610 patients. Surgery 2015;157(2):211-222. https://doi. org/10.1016/j.surg.2014.08.036

14. Kokudo N, Tada K, Seki M, et al. Anatomical major resection versus non-anatomical limited resection for liver metastases from colorectal carcinoma. Am J Surg 2001;181(2):153-159. https://doi. org $/ 10.1016 /$ S0002-9610(00)00560-2

15. Gandy RC, Stavrakis T, Haghighi KS. Short- and long-term outcomes of elderly patients undergoing liver resection for colorectal liver metastasis. Aust N Z J Surg 2016 (epub 30 Oct 2016). https://doi. org $/ 10.1111 /$ ans. 13690

16. Riquet M, Foucault C, Cazes A, et al. Pulmonary resection for metastases of colorectal adenocarcinoma. Ann Thorac Surg 2010;89(2):375-380. https://doi.org/10.1016/j.athoracsur.2009.10.005

17. Iizasa T, Suzuki M, Yoshida S, et al. Prediction of prognosis and surgical indications for pulmonary metastasectomy from colorectal cancer. Ann Thorac Surg 2006;82(1):254-260. https://doi. org/10.1016/j.athoracsur.2006.02.027
18. Pfannschmidt J, Dienemann H, Hoffmann H. Surgical resection of pulmonary metastases from colorectal cancer: A systematic review of published series. Ann Thorac Surg 2007;84(1):324-338. colorectal cancer: A systematic review of publised
https://doi.org/10.1016/jathoracsur.2007.02.093

19. Goonerante D, Gray C, Lim M et al.; Colorectal Lung Metastases Research Group (CRLMRG). Survival outcome in New Zealand after resection of colorectal cancer lung metastases. Aust N Z J Surg Survival outcome in New Zealand after resection of colo
2013;83(12):959-962. https://doi.org/10.1111/ans. 12012

20. Park JH, Lee KH, Han SW et al. The beneficial effect of palliative resection in metastatic colorectal cancer: 10 years of experiences from a single institute. J Clin Oncol 2012;30(Suppl. 4):626. https://doi. org/10.1038/bjc.2013.94

21. Lee KC, Ou YC, Hu WH, Liu CC, Chen HH. Meta-analysis of outcomes of patients with stage IV colorectal cancer managed with chemotherapy/radiochemotherapy with and without primary tumo resection. Onco Targets Ther 2016;9:7059-7069. https://doi.org/10.2147/OTT.S112965

22. Oxenberg J, Papenfuss W, Esemuede I, et al. Multidisciplinary cancer conferences for gastrointestinal malignancies result in measureable treatment changes: A prospective study of 149 consecutive patients. Ann Surg Oncol 2015;22(5):1533-1539. https://doi.org/10.1245/s10434-014-4163-y

23. Leo F, Venissac N, Poudenx M, Otto J, Mouroux J. Multidisciplinary management of lung cancer: how to test its efficacy? J Thorac Oncol 2007;2(1):69-72. https://doi.org/10.1097/JTO.0b013e31802bff56

Accepted 19 September 2017. 